Ann. Biol. anim. Bioch. Biophys., I976, 16 (5), 699-708.

\title{
ÉTUDE COMPARÉE DE L'INFLUENCE DE L'INGESTION DE TROIS FORMES AZOTÉES SIMPLES : UREE, PHOSPHATE DIAMMONIQUE OU URÉE-ACIDE PHOSPHORIQUE SUR QUELQUES CARACTÉRISTIQUES PHYSICO-CHIMIQUES DU CONTENU RUMINAL CHEZ LE MOUTON ADULTE
}

\author{
Rollande DUMON'T et J.-L. TISSERAND \\ avec la collaboration technique de Catherine CoRDELET \\ Labovatoive de Recherches de la Chaive de Zootechnie, \\ Centre de Recherches de Dijon, I. N. R. A., \\ École Nationale Supérieure des Sciences Agronomiques Appliquées, \\ 21016 Dijon Cedex

\section{RÉSUMÉ}

Chez le mouton adulte, l'influence de l'ingestion de trois formes azotées simples, phosphate diammonique (PDA), urée, urée-acide phosphorique, sur certaines caractéristiques physicochimiques du contenu de rumen, est étudiée au cours de deux expériences successives. l'urée.

Dans la première, deux lots de trois moutons ingèrent le phosphate diammonique ou

Dans la seconde, trois lots de trois moutons reçoivent phosphate diammonique, urée ou uréeacide phosphorique.

L'apport de phosphate diammonique ou l'addition d'acide phosphorique à l'urée provoque un abaissement significatif $\mathrm{du} \mathrm{pH}$ du rumen. Cette acidification s'accompagne d'une diminution des concentrations en acides gras volatils totaux, acides acétique et propionique. Le rapport acide acétique/acides gras volatils totaux diminue tandis que le rapport acide propionique/acides gras volatils totaux augmente.

L'ammoniogenèse ne varie pas de façon significative en fonction de la source azotée dans l'essai no 2 mais se révèle plus élevée avec le phosphate diammonique dans l'essai no $\mathbf{I}$.

\section{INTRODUCTION}

La valeur azotée réelle de l'urée ajoutée à une ration pour ruminants dépend de la fraction d'azote qui, sous forme d'ammoniac, diffuse hors du rumen et devient donc inutilisable pour les synthèses bactériennes.

Le $\mathrm{pH}$ du contenu de rumen influant sur la vitesse de passage de l'ammoniac 
à travers la paroi (BLOOMFIEL,D, I963), l'acidification du contenu ruminal peut constituer un moyen de limiter cette diffusion. Un certain nombre d'auteurs étudient l'utilisation, dans ce but, de sels d'ammonium (phosphates ammoniacaux), ou encore l'addition d'acides minéraux (acide phosphorique par exemple) à des rations contenant de 1'urée. En pratique, ces phosphates se révèlent beaucoup moins toxiques pour les ruminants que l'urée (RUSSEI, et al., I962 ; OL,TJEN et al., I963 ; RICHARDson, I966) ; leur appétibilité et leur utilisation digestive et métabolique restent très comparables à celles de l'urée (OLtrJEn et al., 1963; Johnson et McLuRE, 1963; R. CoMPÈRE, I969), toutefois leur taux d'incorporation dans la ration ne doit pas être trop élevé.

Pour compléter les résultats obtenus, en particulier en ce qui concerne l'intensité des fermentations et la production d'acides gras volatils, nous avons effectué deux essais destinés à :

- comparer les variations du $\mathrm{pH}$ des contenus de rumen de mouton recevant une partie de l'azote de leur ration sous forme de phosphate diammonique (PDA), urée, urée-acide phosphorique (urée $+\mathrm{H}_{3} \mathrm{PO}_{4}$ ) ;

- estimer les activités de la flore bactérienne avec ces différentes sources azotées en mesurant les teneurs en ammoniac $\left(\mathrm{NH}_{3}\right)$ et acides gras volatils (AGV) des jus de rumen.

\section{MATÉRIEL ETT MÉTHODES}

\section{I. - Animaux et aliments}

Essai $n^{\mathrm{o}} 1$.

Six moutons adultes de race Lacaune, castrés, pesant en moyenne $47 \mathrm{~kg}$, porteurs d'une canule permanente du rumen, sont répartis en 2 lots homogènes. Pour chaque animal, la ration journalière distribuée en 2 repas est composée de :

- I $\mathrm{kg}$ de mais plante entière déshydraté et compacté ayant la composition suivante : MS = 91,4 p. I00; $\mathrm{MM}=4$,I p. I00; $\mathrm{CB}=15,6$ p. I00; $\mathrm{MAT}=7,5$ p. I00;

- $50 \mathrm{~g}$ de complément minéral vitaminisé ;

- I supplément azoté :

Lot I : I $5 \mathrm{~g}$ d'urée dosant $43,5 \mathrm{p}$. Ioo d'azote et apportant $6,52 \mathrm{~g}$ d'azote ;

Lot $2: 3^{\mathrm{T}} \mathrm{g}$ de phosphate diammonique $\left(\mathrm{NH}_{4}\right)_{2} \mathrm{HPO}_{4}$, dosant $2 \mathrm{I}, 2 \mathrm{p}$. 100 d'azote et apportant $6,57 \mathrm{~g}$ d'azote.

Ces deux rations sont donc isoénergétiques et isoazotées mais ont des teneurs en phosphore différentes, l'apport de phosphore par le PDA étant relativement important.

Essai no 2 .

Neuf moutons adultes de race Ile-de-France, castrés, pesant en moyenne $75 \mathrm{~kg}$, munis d'une canule permanente du rumen, sont répartis en 3 lots homogènes. Ils reçoivent chaque jour :

- de la paille à volonté ;

- I,300 $\mathrm{kg}$ de mais plante entière déshydraté et compacté ayant la composition suivante : MS $=92, \mathrm{I}$ p. I00; $\mathrm{CB}=19,8$ p. I00; $\mathrm{MM}=4,2$ p. I00; MAT $=8,2$ p. I00;

- I supplément azoté différent selon le lot:

Lot I : $18 \mathrm{~g}$ d'urée à $43,5 \mathrm{p}$. I0o d'azote, soit $7,83 \mathrm{~g}$ d'azote :

Lot 2: $3^{8} \mathrm{~g}$ de PDA à $2 \mathrm{I}, 2 \mathrm{p}$. Ioo d'azote, soit $8,06 \mathrm{~g}$ d'azote ;

Lot 3 : $18 \mathrm{~g}$ d'urée à $43,5 \mathrm{p}$. Ioo d'azote et $\mathrm{I} 8,7 \mathrm{ml} \mathrm{d} \mathrm{H}_{3} \mathrm{PO}_{4}(85 \mathrm{p}$. Ioo) dilué ;

- I complément minéral de composition variable selon le lot permettant d'égaliser les apports en phosphore dans les trois types de ration.

Cette ration est distribuée en 2 repas. Dans le cas du lot $3,250 \mathrm{ml}$ de la solution acide sont introduits par la canule au début de chaque repas. 


\section{II. - Dispositif expérimental}

La période d'adaptation aux régimes est de 3 semaines (essai no 1 ) et 5 semaines (essai no 2 ).

Pour chaque essai, quatre séries de prélèvements sont effectuées à 5, 7,2 jours d'intervalle (essai $n^{\circ} I$ ), une semaine d'intervalle (essai $n^{\circ} 2$ ).

Les prélèvements de contenu de rumen sont faits à travers la canule au moyen d'une pompe aspirante munie d'un tube de polyvinyl souple.

Le premier prélèvement a lieu à jeun (temps o) et les suivants $I h, I h 30,2 h 30$, $3 \mathrm{~h} 30,4 \mathrm{~h} 30,5 \mathrm{~h} 30,7 \mathrm{~h}$ 30 après le début du repas du matin (essai $\mathrm{n}^{\circ} \mathrm{I}$ ), I $\mathrm{h}, 2 \mathrm{~h}, 3 \mathrm{~h}, 4 \mathrm{~h}, 5 \mathrm{~h}$, 7 h (essai no 2 ).

Les jus de rumen sont analysés en vue de la détermination :

- $\mathrm{du} \mathrm{pH}$,

- des bases azotées volatiles exprimées en $\mathrm{N}-\mathrm{NH}_{3}$ par microdiffusion (ConwaY, 1950),

- des AGV par chromatographie phase gazeuse sur colonne de verre de $2 \mathrm{~m}$ de long et $3 \mathrm{~mm}$ de diamètre interne. Le support chromosorb W 80-Ioo est imprégné de $25 \mathrm{p}$. Ioo de NPG.t (N pentyl glycol adipate) et de 2 p. roo d'acide phosphorique.

\section{III. - Analyse statistique des résultats}

Les deux essais sont traités comme essai factoriel à deux facteurs contrôlés avec répétitions (test de $\mathrm{F}$ et calcul de la plus petite différence significative).

Le premier facteur est la supplémentation azotée :
2 variantes
essai $n^{0} \mathrm{I}$.
3 variantes
essai $n^{0} 2$.

Le deuxième facteur est le temps de prélèvement par rapport au repas du matín :

$\begin{array}{ll}8 \text { variantes } & \text { essai } n^{0} I \\ 7 \text { variantes } & \text { essai } n^{0} 2 .\end{array}$

Les quatre répétitions correspondent aux séries de mesures effectuées durant 4 semaines différentes. Chaque résultat est la moyenne des trois moutons du même lot. Le seuil de signification retenu est $\mathbf{P}<0,05$.

\section{RÉSULTATS}

\section{A. - Evolution postprandiale du $p H$ ruminal}

Les $\mathrm{pH}$ mesurés avec le régime $\mathrm{PDA}$ (fig. $\mathrm{I})$ sont inférieurs $(\mathrm{P}<0,05)$ à ceux obtenus avec l'urée. De même, avec le mélange urée- $\mathrm{H}_{3} \mathrm{PO}_{4}$, les $\mathrm{pH}$ restent plus faibles qu'avec les deux autres sources azotées.
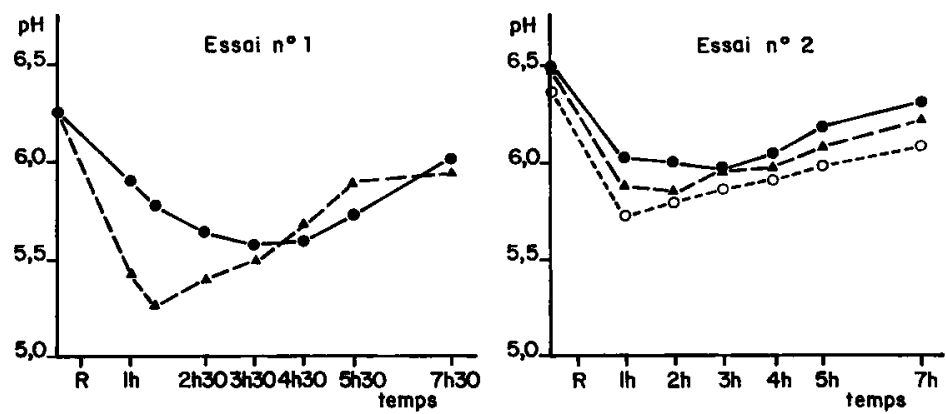

FIc. I. - Évolution postprandiale du pH ruminal

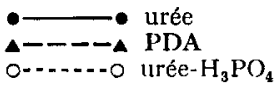


L'évolution de l'acidité dans le temps est la même quelle que soit la source azotée ; en effet, 1'analyse statistique montre que l'interaction temps de prélèvement-source azotée n'est pas significative.

Cette évolution peut être schématisée de la manière suivante :

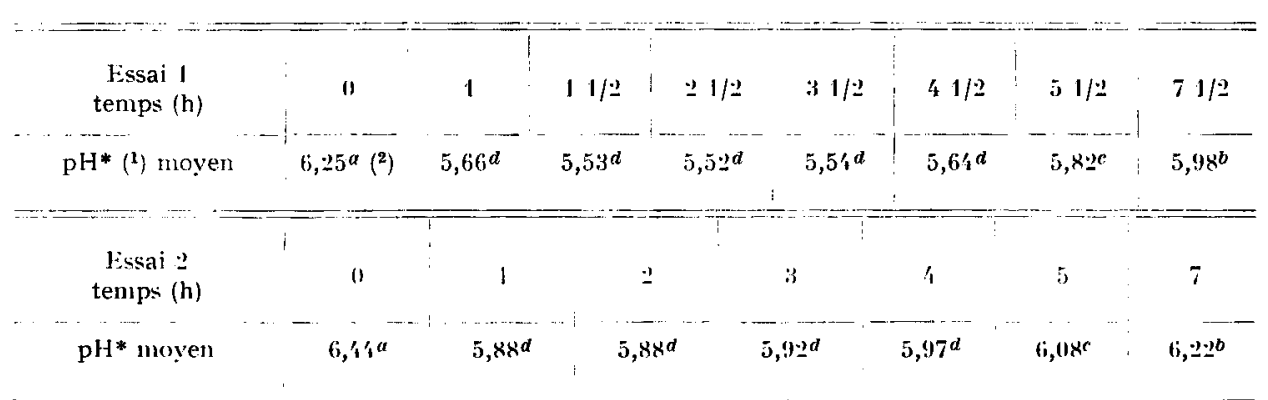

(1) * Seuil de signification $\mathrm{P}<\mathbf{0 , 0 5}$.

(2) $a, b, c \ldots$ les valeurs suivies d'une même lettre ne sont pas significativernent différentes; les valeurs nơı suivies d'une même lettre sont significativement différentes et se classent dans l'ordre $a>b>c \ldots$

Le $\mathrm{pH}$ diminue après le repas, reste sensiblement constant pendant les 4 heures (essai 2) ou les 5 heures (essai I) qui suivent, puis augmente ensuite.

\section{B. - Evolution postprandiale}

de la teneur en bases azotées volatiles $\left(\mathrm{N}-\mathrm{NH}_{3}\right)$ du jus de rumen (fig. 2)

Dans l'essai no $\mathrm{I}$, la teneur en $\mathrm{NH}_{3}$ avec le PDA est supérieure $(\mathrm{P}<0,05)$ à celle obtenue avec l'urée, cela n'est pas confirmé par l'essai $n^{\circ} 2$.

L'effet temps de prélèvement est, par contre, significatif dans les deux essais.

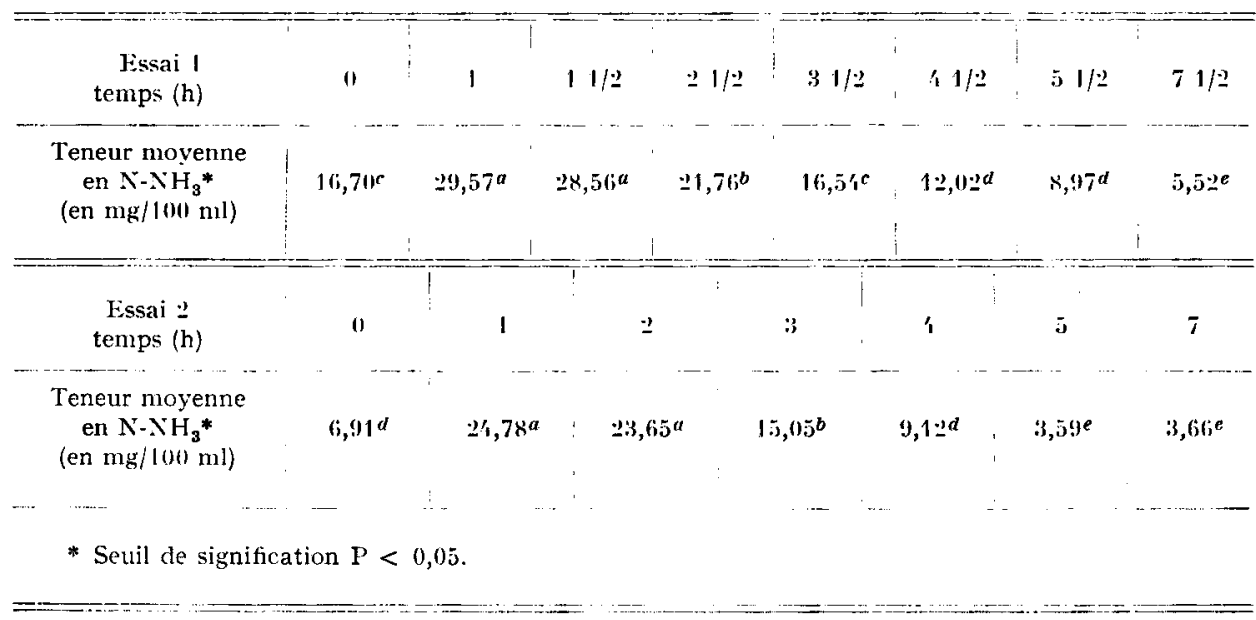


Le taux de $\mathrm{N}-\mathrm{NH}_{3}$ passe par un maximum I à 2 heures après le repas, quelle que soit la source azotée ; les évolutions dans le temps des teneurs en $\mathrm{N}-\mathrm{NH}_{3}$ sont semblables pour les différentes rations (facteur interaction non significatif).
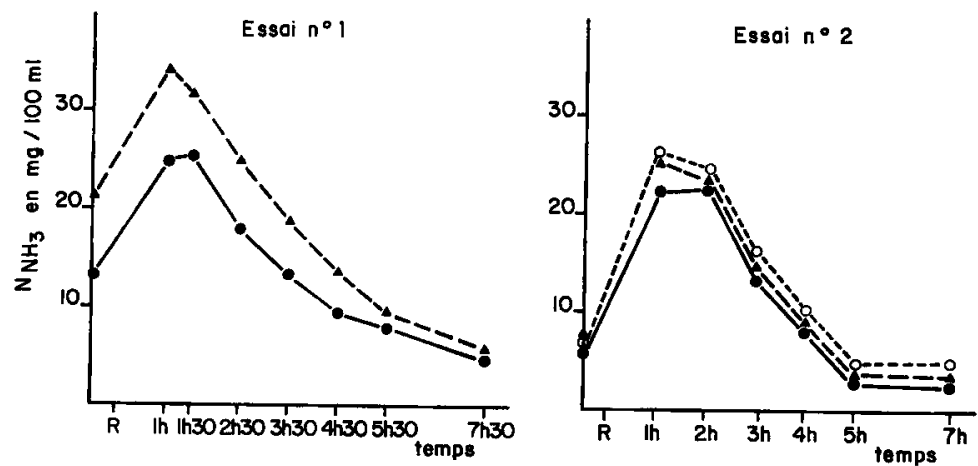

FIG. 2. - Évolution postprandiale

des teneurs en bases azotées volatiles $\left(\mathrm{N}-\mathrm{NH}_{3}\right)$ des contenus de rumen

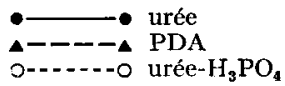

\section{C. - Évolution postprandiale}

des concentrations en $A G V$ du jus de rumen (fig. 3 et 4 )

Les concentrations en AGV totaux sont significativement inférieures dans le cas du PDA comparativement à l'urée. Par contre, il n'y a pas de différence significative entre le PDA et le mélange urée- $\mathrm{H}_{3} \mathrm{PO}_{4}$.

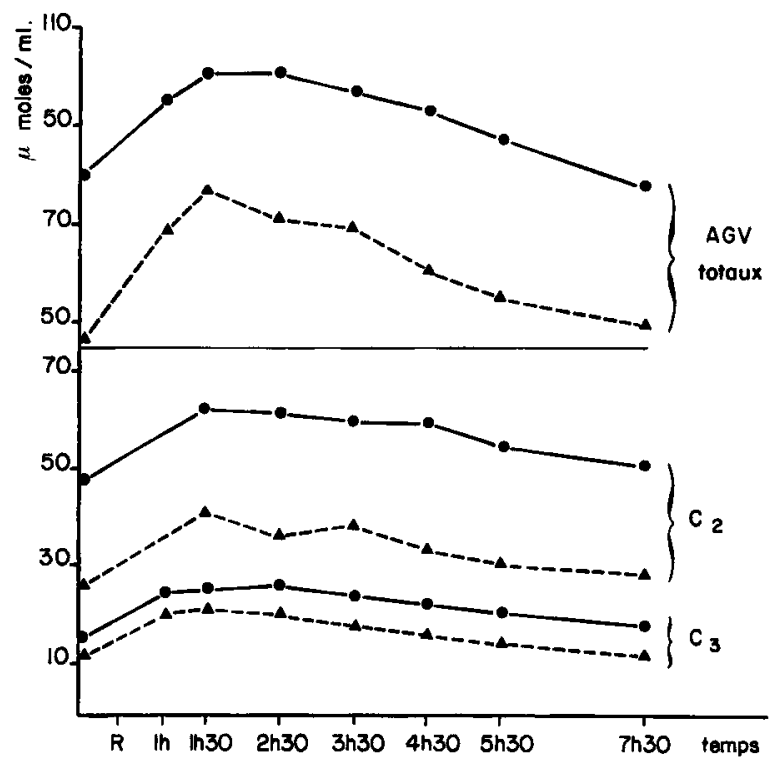

FIr. 3. - Évolution postprandiale des concentrations en A GV totaux, acide acétique, acide propionique (Essai $\mathrm{n}^{0} \mathrm{I}$ )

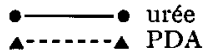


La concentration en $A G V$ totaux passe par un maximum I à 4 h après le début du repas, quelle que soit la source azotée. Les valeurs obtenues aux différents temps de prélèvement se classent comme suit :

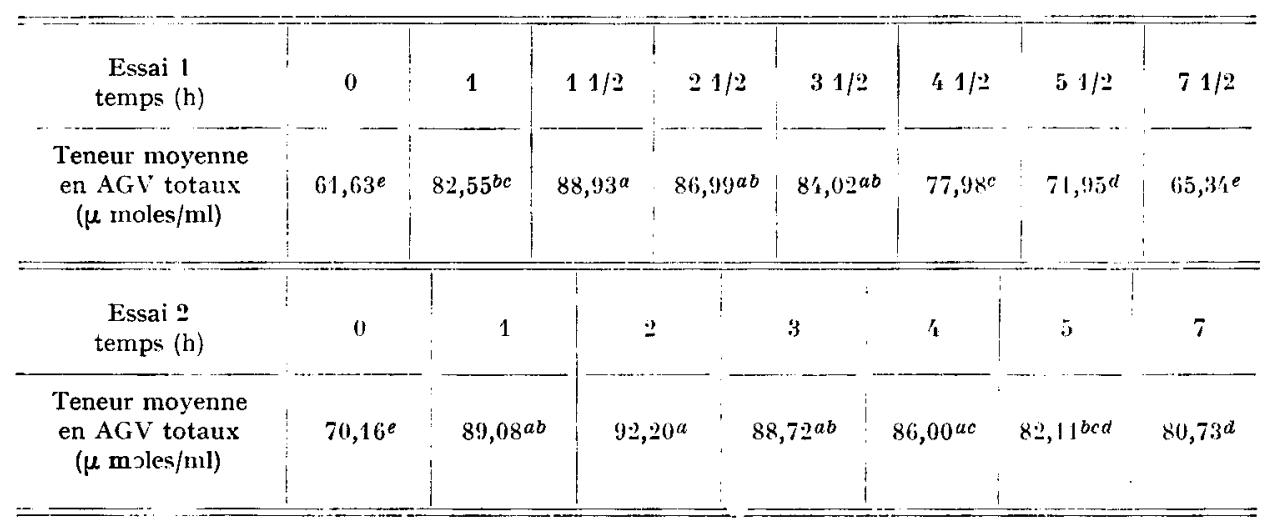

Ceci se retrouve au niveau des acides acétique et propionique dont les teneurs sont significativement inférieures avec le PDA comparativement à l'urée pour les deux essais. Par contre, les teneurs ne sont pas différentes entre acide-urée et PDA.

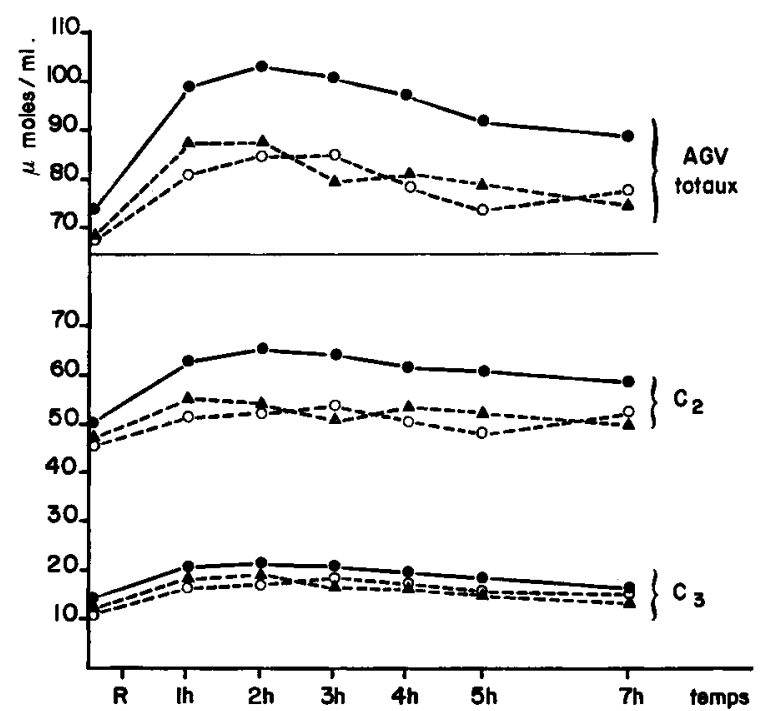

FIG. 4. - Evolution postprandiale des concentrations en $A G Y$ totaux, acide acétique, acide propionique (Essai $\mathrm{n}^{\circ} 2$ )

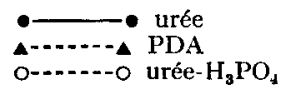

Cette diminution de la concentration en AGV totaux, acide acétique et acide propionique, s'accompagne également d'une modification des proportions molaires des différents acides (tab1. I et 2). Avec le PDA, le rapport acide acétique/AGV 
totaux $\times$ Ioo est significativement inférieur comparativement à l'urée dans l'essai $n^{\circ} I$; on ne note pas de différence dans l'essai $n^{\circ} 2$. Le rapport acide propionique/ $A G V_{T} \times$ roo est significativement plus élevé avec le PDA comprativement à l'urée.

TABLEAU I

Evolution postprandiale des proportions molaires des acides acétique et propionique (Essai no r)

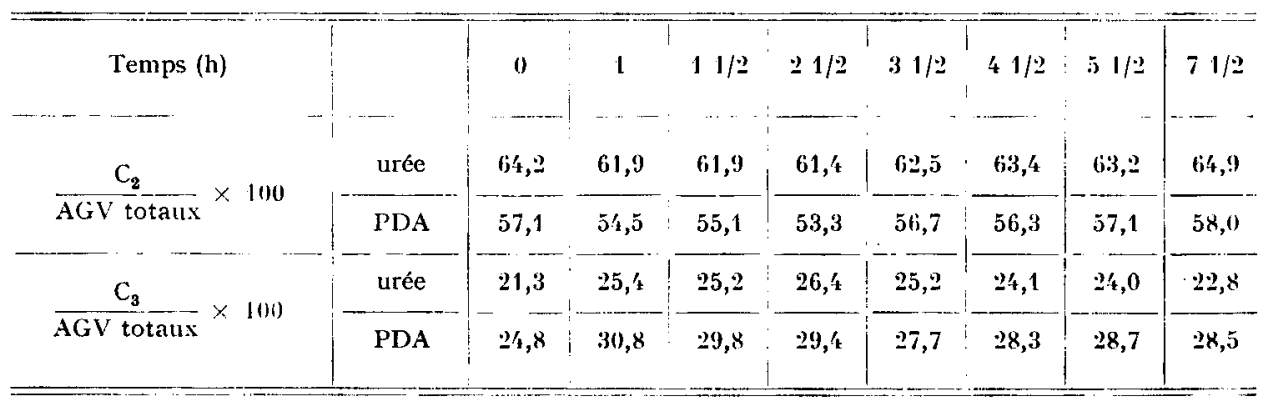

TABLEAU 2

Evolution postprandiale des proportions molaires des acides acétique et propionique (Essai no 2)

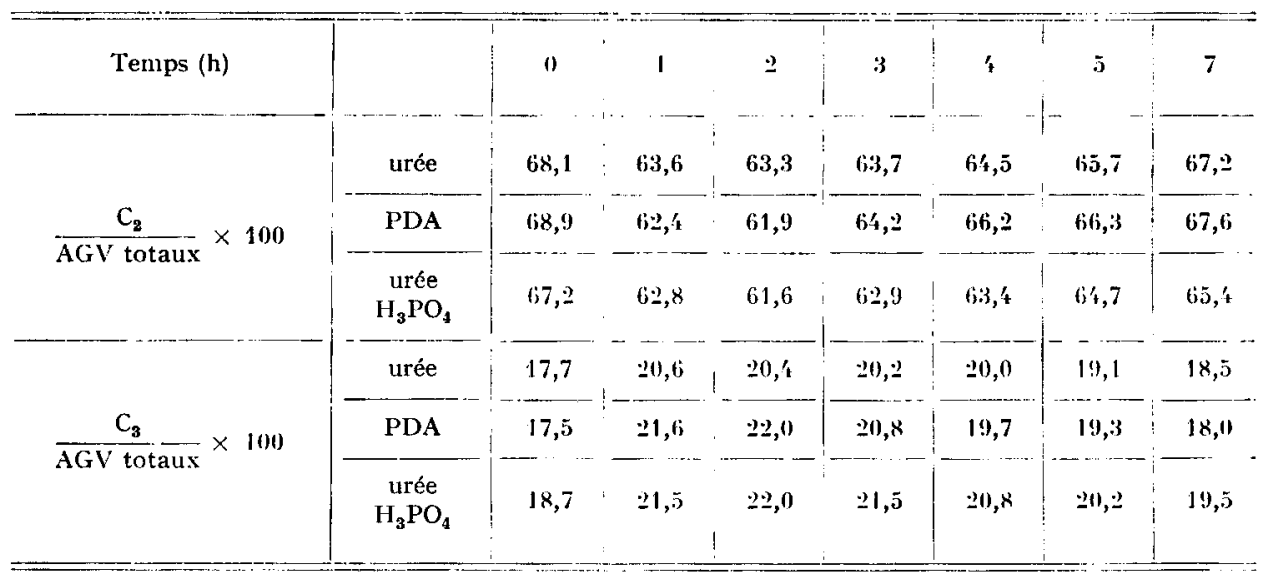

Au cours du I er essai, les taux acide acétique/AGV ${ }_{\mathbf{T}}$ et acide propionique/AGVT sont invariables au cours du temps; par contre, dans le $2^{\text {e }}$ essai, le rapport acide acétique/AGV $/ \mathrm{Al}_{\mathbf{T}}$ diminue puis augmente, le rapport acide propionique/AGV $\mathrm{AV}_{\mathbf{T}}$ volue en sens inverse.

\section{DISCUSSION}

Il est relativement difficile d'interpréter les résultats de ces deux essais puisqu'i1 s'agit toujours de concentrations en ions $\mathrm{H}^{+}$, ammoniac et acides gras, et non des quantités totales des différents métabolites produits. 
Chaque concentration est le résultat de trois phénomènes : les activités fermentaires, la vitesse de transit vers l'intestin, la vitesse d'absorption à travers l'épithélium ruminal.

L'apport de $\mathrm{PDA}$ ou l'addition $\mathrm{d}^{\prime} \mathrm{H}_{3} \mathrm{PO}_{4}$ à l'urée provoque une diminution du $\mathrm{pH}$ postprandial. Ce résultat confirme les travaux de RUSSEI et al. (I962), OLTJEN et al. (I963), PEREZ et al. (I967). MAROADI (I972) signale également une diminution du $\mathrm{pH}$ dans les heures qui suivent le repas lorsqu'il distribue de l'urée-phosphate à des bovins ; cependant, au bout de $\mathrm{I} 8$ semaines d'expérience, il n'observe pratiquement plus cette chute du $\mathrm{pH}$.

Cette acidification du contenu ruminal serait due aux deux premières acidités de l'acide phosphorique, ou, dans le cas du PDA, au fait que le phosphate d'ammonium est un sel d'acide faible et de base faible.

Parallèlement à cette baisse de pH avec le PDA, l'essai no I seul montre une augmentation de la teneur en bases azotées volatiles $\left(\mathrm{N}-\mathrm{NH}_{3}\right)$, signalée également dans les travaux de Perez (I967). Par contre, dans l'essai no 2 , il n'y a pas de différence des ammoniogenèses selon les trois sources azotées. OLTJEN et al. (I968) mettent en évidence un taux maximum de $\mathrm{N}-\mathrm{NH}_{3}$ significativement supérieur avec l'urée comparativement à l'urée-phosphate.

Il est possible de penser que la vitesse de diffusion de l'ammoniac à travers la paroi du rumen est diminuée avec des rations PDA ou acide phosphorique. En effet, les teneurs maxima de $\mathrm{N}-\mathrm{NH}_{3}$ et d'urée du sang périphérique sont généralement plus faibles avec les phosphates ammoniacaux qu'avec l'urée (Russel, et al., I962 ; Johnson et McCluRE, I964. PEREz et al., I967).

Cet abaissement du $\mathrm{pH}$ peut être mis en relation avec la diminution des activités fermentaires mesurées par la diminution des concentrations en AGV. Le pH le plus bas enregistré dans nos deux essais atteint la valeur 5,28. Or, d'après ESDALE et SATTER (I972), lorsque le $\mathrm{pH}$ diminue de 6,8 à 6,2, la production d'AGV n'est pas modifiée; par contre de 6,2 à 5,6 , le taux d'AGV totaux décroît de $25 \mathrm{p}$. Ioo, le rapport acide acétique/acide propionique diminue également.

Ce sont surtout les bactéries cellulolytiques et amylolytiques qui utilisent 1'ammoniac. Une diminution de l'activité cellulolytique peut alors s'accompagner d'une diminution de 1'utilisation de l'ammoniac, d'où son accumulation (le $\mathrm{pH}$ limitant la diffusion). Cela pourrait expliquer les plus fortes teneurs en $\mathrm{N}-\mathrm{NH}_{3}$ enregistrées avec le PDA.

Enfin, les faibles teneurs en AGV enregistrées avec les rations PDA peuvent être mises en relation avec le $\mathrm{pH}$ du rumen; en milieu acide, l'absorption des AGV est plus rapide.

\section{CONCLUSION}

Le PIAA ou l'acide phosphorique acidifient le contenu ruminal et limitent ainsi la vitesse de diffusion de l'ammoniac hors du rumen. Cela n'implique pas obligatoirement une diminution de la quantité totale d'azote perdue par cette voie. Dans la pratique, les rétentions azotées mesurées avec le PDA ou l'urée phosphate ne sont pas meilleures que celles obtenues avec 1'urée (OLTJEN et al., I963; JoHnson et McClURE, Ig63-I964). 
L'apport de phosphate diammonique ou l'addition d'acide phosphorique à l'urée provoque une baisse de la concentration en acides gras volatils totaux (acide acétique et acide propionique notamment). Le rapport acide acétique/acides gras volatils totaux diminue tandis que le rapport acide propionique/acides gras volatils totaux augmente.

Étant donné la sensibilité des métabolismes bactériens aux variations de $\mathrm{pH}$, de telles sources d'azote ne sont peut-être pas à rechercher. Par contre, le phosphate diammonique en tant que source de phosphore pourrait se révéler intéressant dans la mesure où l'utilisation de son phosphore est satisfaisante et où il ne pose pas de problème d'appétence.

Reçu pour publication en fétrier 1976.

\section{SUMMARY}

COMPARATIVE STUDY OF NITROGEN INGESTED IN THREE SIMPLE FORMS : UREA, DIAMMONIC PHOSPHATE OR PHOSPHORIC ACID-UREA. EFFECT ON SOME PHYSICO-CHEMICAI CHARACTERISTICS OF ADULT SHEEP RUMEN CONTENT

Nitrogen is ingested by adult sheep in three simple forms : diammonic phosphate (PDA), urea and phosphoric acid-urea. Its effect on some physico-chemical characteristics of rumen content is studied during two successive experiments.

In the first, two lots of three sheep eat diammonic phosphate or urea.

In the second, three lots of sheep are given diammonic phosphate, urea or phosphoric acidurea.

The diammonic phosphate supply or addition of phosphoric urea-acid causes a significant drop in rumen $\mathrm{pH}$. This acidification is accompanied by a decrease in total volatile fatty acids and acetic and propionic acids. The ratio acetic acid/total volatile fatty acids decreases, while the ratio propionic acid/total volatile fatty acids increases.

Ammoniogenesis does not vary significantly in relation to nitrogen source in trial 2 , but is higher with diammonic phosphate in trial $\mathrm{I}$.

\section{RÉFÉRENCES BIBLIOGRAPHIQUES}

Blackburn Th., 1964. Nitrogen metabolism in the rumen. In Physiology of digestion in the ruminant ed. by R. W. DOugherty London, 322-334.

Compère R., I969. Valeur comparée des phosphates monoammonique et diammonique comme sources d'azote et de phosphore alimentaires chez le Mouton. Bull. Rech. A gron. Gemblontx, 4, 339-367.

Conway F. J., 1962. Micro-diffusion Analysis and Volumetric Error., ed. 5 Crosby Lockwood and Son, London.

Esdale Wt., Satter L. D., I972. Manipulation of ruminal fermentation. IV. Effect of altering ruminal $\mathrm{pH}$ on volatile fatty acid production. J. Dairy Sci., 55, 964-969.

Johnson R. R., McClure K. E., I963. In vitro and in vivo, studies on the adaptation of sheep to biuret. J. Anim. Sci., 22, II23.

JohNSON R. R., MCCluRe K. E., I964. In vitro and in vivo comparisons on the utilization of urea, biuret and diammonium phosphate by sheep. J. Anim. Sci., 23, 208-2r3.

Maroadi A., Piva G., Rostelli A., Caprioli G., 1972. Urea-phosphate in the feeding of young cattle. Nutr. abstr. 43, 6879.

Oltjen R. R., Waller G. R., Nelson A. B., Thllman A. D., ig63. Ruminant studies with diammonium phosphate and urea. J. Anim. Sci., 22, 36-4I. 
OLtJeN R. R., Styter L. L., KozAK A. S, E. E. Williams J. R., Ig68. Evaluation of urea, urea phosphate, biuret and uric acid as NPN sources for cattle. J. Nutr., 94, I93-202.

Perez C. B, WARNER R. G., Loosli J. K., I967. Evaluation of urea-phosphate as a source of nitrogen and phosphorus for ruminants. $J$. Anim. Sci, 26, 810-8Ig.

Russell E. L., Hale W. H., Farris Hubbert J, R., 1962. Evaluation of diammonium phosphate as a source of nitrogen for ruminants. J. Anim. Sci., 21, 523-526.

Richardson, I966, cité par BNA IT 4I8 BO, т968. L'utilisation des phosphates d'ammonium dans les aliments composés pour ruminant et les ensilages. 